\title{
PromISR-6, a Guanabenz Analogue, Improves Cellular Survival in an Experimental Model of Huntington's Disease
}

(Manuscript ID:cn-2019-00185d)

\section{SUPPLEMENTAL METHODS}

\section{Genome-wide Gene Expression Analysis}

Genome-wide gene expression or microarray analyses (Genechip ${ }^{\circledR}$ Rat gene 1.0 ST array, Affymetrix, Cleveland, OH, USA) were performed using RNA extracted from PC12 cells expressing GFP-mHtt-74Q, separated into four groups with 3 replicates for each group: non-induced and DMSO-treated, doxycycline-induced and DMSO-treated, doxycyclineinduced and $10 \mu \mathrm{M}$ Compound 3-treated and doxycycline-induced and $10 \mu \mathrm{M}$ PromISR 6treated for $48 \mathrm{hr}$. Signals across all microarrays were log transformed (base 2) and quantile normalized after background correction via the robust multichip averaging (RMA) method $^{l}$ in Expression Console (Affymetrix, USA). Principal components analysis (PCA) was conducted via the princomp package in $\mathrm{R}$ to identify sample outliers. Statistical significance of differentially expressed genes was ascertained via a regularized t-test based on the Bayesian statistical framework ${ }^{2}$. False positives due to multiple testing was controlled by the false discovery rate (FDR). The magnitude of gene over-expression or under-expression was quantified by estimating the difference in the $\log 2$ average signals between treated and control groups. Genes with a FDR $\leq 1 \%$ and absolute fold-change $\geq 1.5$-fold were defined as differentially expressed. To identify biochemical pathways impacted by the differential gene expression, we analyzed microarray data via gene-set enrichment analysis (GSEA) ${ }^{3}$ of the Reactome pathway repository. Pathways with a FDR $\leq 5 \%$ were considered to be significantly enriched. The sharing of genes among top regulated pathways (FDR $\leq 5 \%$ ) was visualized via the EnrichmentMap application in Cytoscape, where pathways with an overlap coefficient $\geq 0.5$ 
were considered to be overlapping in gene content. The MIAME compliant microarray data have been deposited in the National Center for Biotechnology Information (NCBI) Gene Expression Omnibus (GEO) repository, under accession number GSE119557.

\section{Cell Viability using PrestoBlue}

PC12 cells expressing GFP-mHtt-74Q (7,000 cells/well) seeded in 96 well plates (Black, 655096, Greiner bio-one), were treated with DMSO (Vehicle) or PromISR-6 over a wide range of concentrations for $48 \mathrm{hr}$. The PrestoBlue reagent (A13261, ThermoFisher scientific) was added to each well in a 1:10 dilution and incubated for 30 minutes at $37^{\circ} \mathrm{C}$ with $5 \% \mathrm{CO} 2$ according to manufacturer's instructions. Fluorescence was measured with $\lambda \mathrm{ex}=560$ $\mathrm{nm}$ and $\lambda \mathrm{em}=590 \mathrm{~nm}$ using Tecan Infinite M200 microplate reader. The background fluorescence was subtracted from all the values and percent viability was calculated for cells exposed to PromISR-6 relative to the vehicle (DMSO). 


\section{SUPPLEMENTAL FIGURES}

\section{Figure S1}

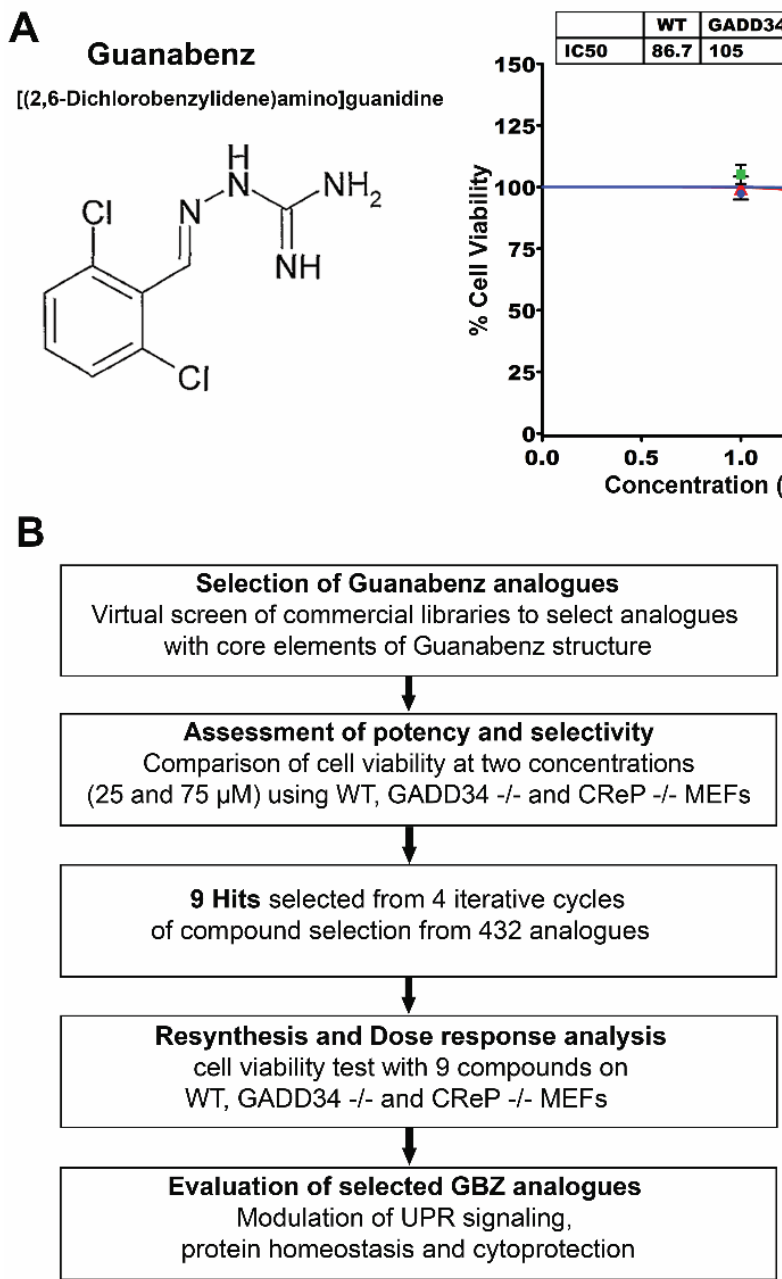

\section{Supplemental Figure S1: Screening of Guanabenz Analogues}

Panel A shows the structure of Guanabenz and its dose-dependent cytotoxicity in WT, GADD34-/- or CReP-/- MEFs treated for $48 \mathrm{hr}(\mathrm{n}=3)$. The $\mathrm{IC}_{50}$ for each cell is shown in the boxes. Error bars indicate \pm s.e.m. Panel B shows the work plan for screening and analysis of Guanabenz analogues. 


\section{Figure S2}

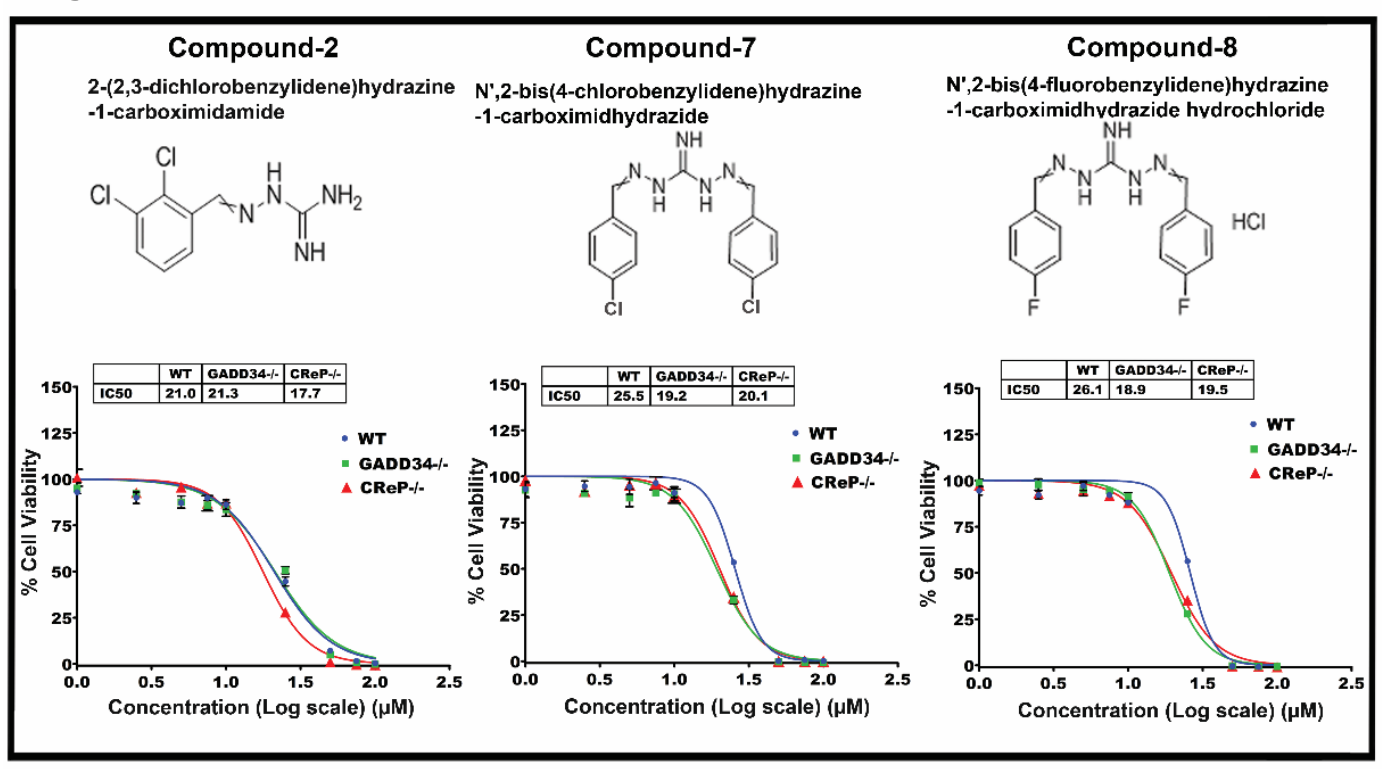

\section{Supplemental Figure S2: Cytotoxicity of Group 1 Guanabenz analogues}

Dose response curves for cytotoxicity of WT, GADD34-/- and CReP-/- MEFs (n=3) to Compounds 2, 7 and 8, shown with their chemical structures, are displayed $(n=3)$. $\mathrm{IC}_{50}$ values for each compound with each cell are shown in the boxes. Error bars indicate \pm s.e.m. 


\section{Figure S3}

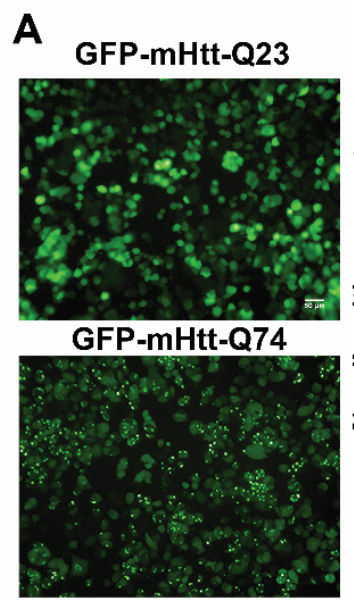

C

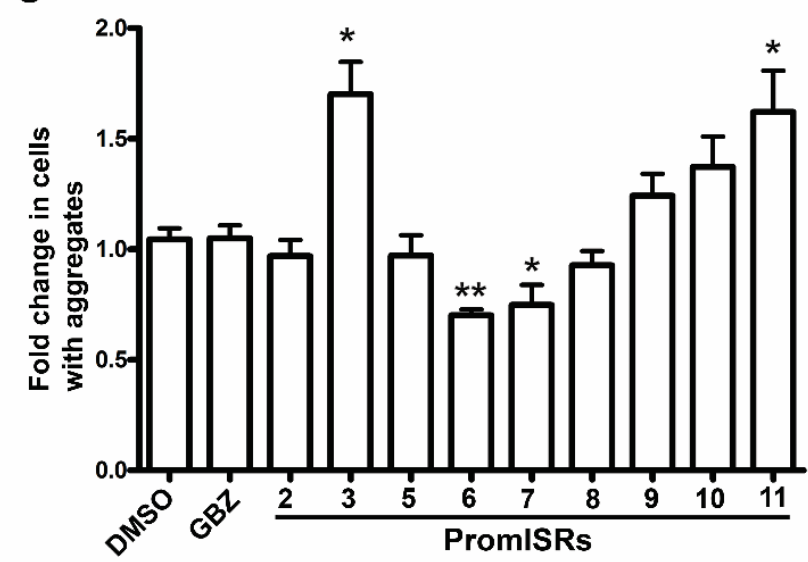

B

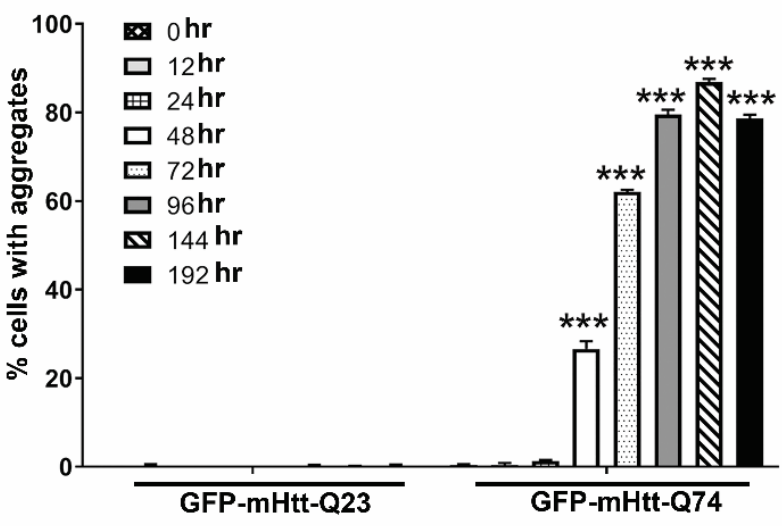

D

Supplemental Figure S3: Aggregation of GFP-mHtt-74Q in PC12 Cells in the Presence of Guanabenz Analogues

Panel A shows representative images of PC12 cells induced to express GFP-Huntingtin with either 23Q (control, non-aggregation prone Huntingtin) or 74Q repeats (mutant, aggregationprone Huntingtin) following the addition of doxycycline to culture media for $72 \mathrm{hr}$. Scale bar represents $50 \mu \mathrm{m}$. Panel B shows a bar graph of the percentage of cells with GFP-Htt-74Q aggregates over time $(\mathrm{n}=2)$. Error bars indicate \pm s.e.m $(* * * \mathrm{p}$-value $\leq 0.001)$. Panel $\mathrm{C}$ shows a bar graph of fold-change in cells with GFP-mHtt-74Q aggregates exposed to DMSO (vehicle), Guanabenz or analogues, shown by their compound numbers $(10 \mu \mathrm{M})$ for $72 \mathrm{hr}$ (three independent experiments were performed in duplicates). Error bars indicate \pm s.e.m (** p-value $\leq 0.01$ and * $p$-value $\leq 0.05$ ). Panel D shows a bar graph of the change in cells with GFP-mHtt$74 \mathrm{Q}$ aggregates exposed to DMSO (vehicle) and increasing Sephin 1 concentrations for $72 \mathrm{hr}$. 


\section{Figure S4}

A
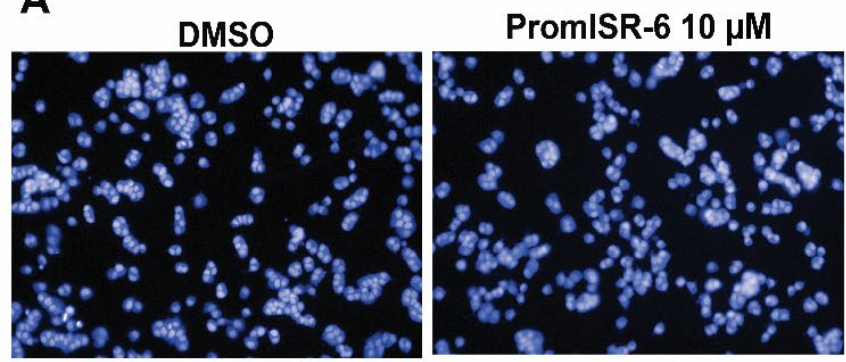

PromISR-6 $25 \mu \mathrm{M}$
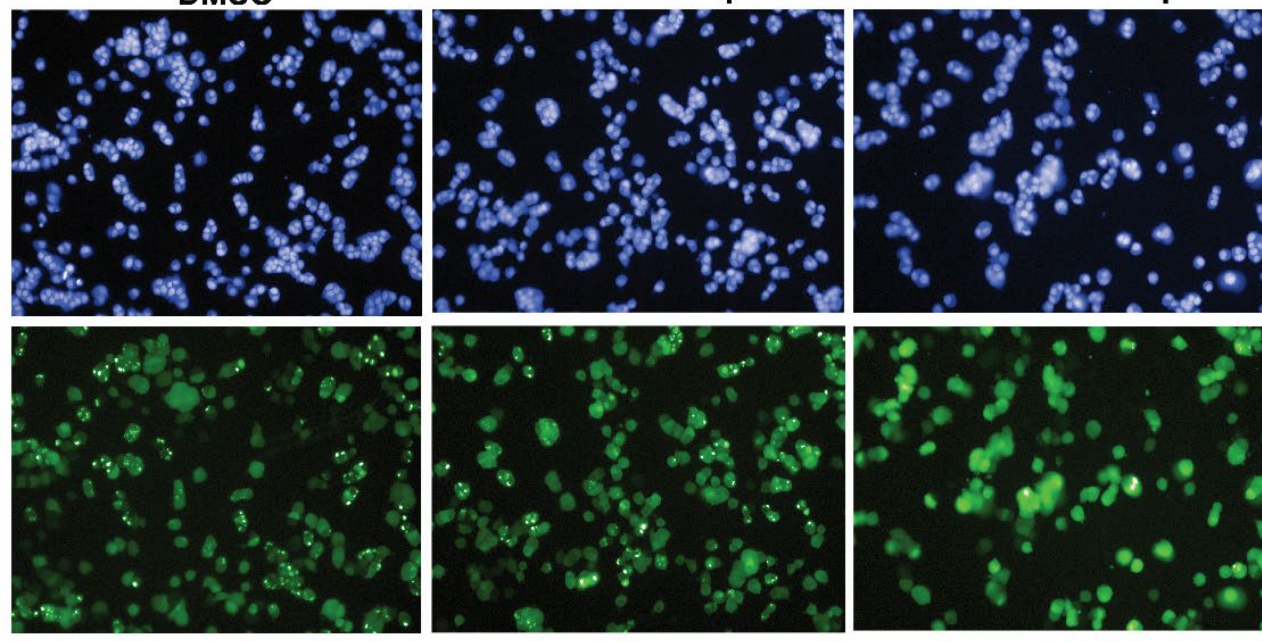

B

C

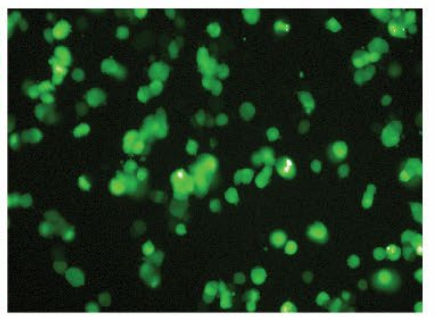

D
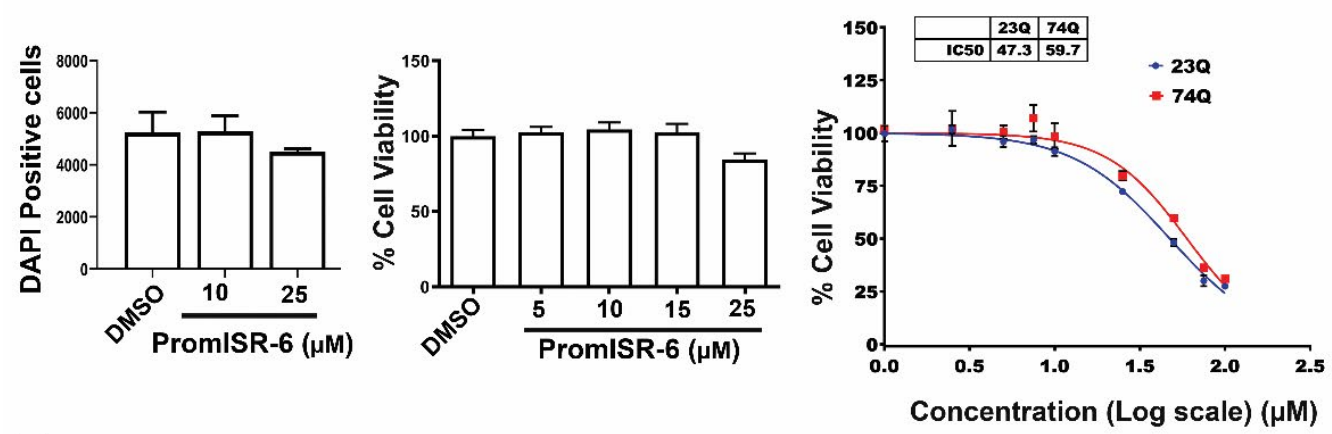

$\mathbf{E}$

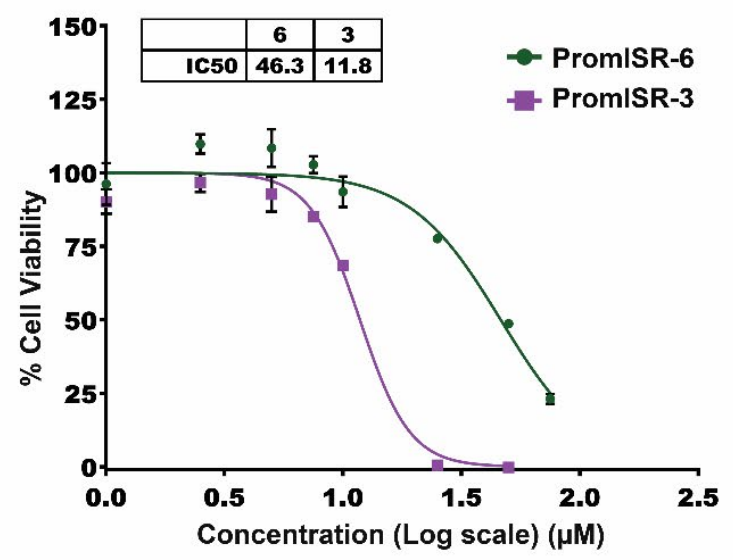

Supplemental Figure S4: Cytotoxicity of GFP-mHtt-74Q PC12 Cells with Guanabenz Analogues

Panel A shows representative images of PC12 cells, expressing GFP-mHtt-74Q induced by doxycycline, in DMSO (vehicle), PromISR-6 for $48 \mathrm{hr}$. Panel B shows the bar graph of number of DAPI positive cells expressing GFP-mHtt-74Q following treatments described for Panel A. Panel C shows the bar graph of percentage of viability of cells expressing GFP-mHtt-74Q 
exposed to increasing PromISR-6 concentrations for $48 \mathrm{hr}$ using Prestoblue as described in Supplemental Methods ( $n=3$ ). Panel D shows the dose response curves for cytotoxicity of cells expressing GFP-mHtt-74Q and GFP-mHtt-23Q to PromISR-6 using CellTiter Glo luminescence $(n=3)$ as described in Methods. $\mathrm{IC}_{50}$ values for PromISR-6 with each cell are shown in the box. Panel E shows the dose response curves for cytotoxicity of cells expressing GFP-mHtt-74Q to PromISR-6 and PromISR-3 for $72 \mathrm{hr}(\mathrm{n}=3)$. IC 50 values are shown in the box. Error bars indicate \pm s.e.m.

\section{Figure S5}
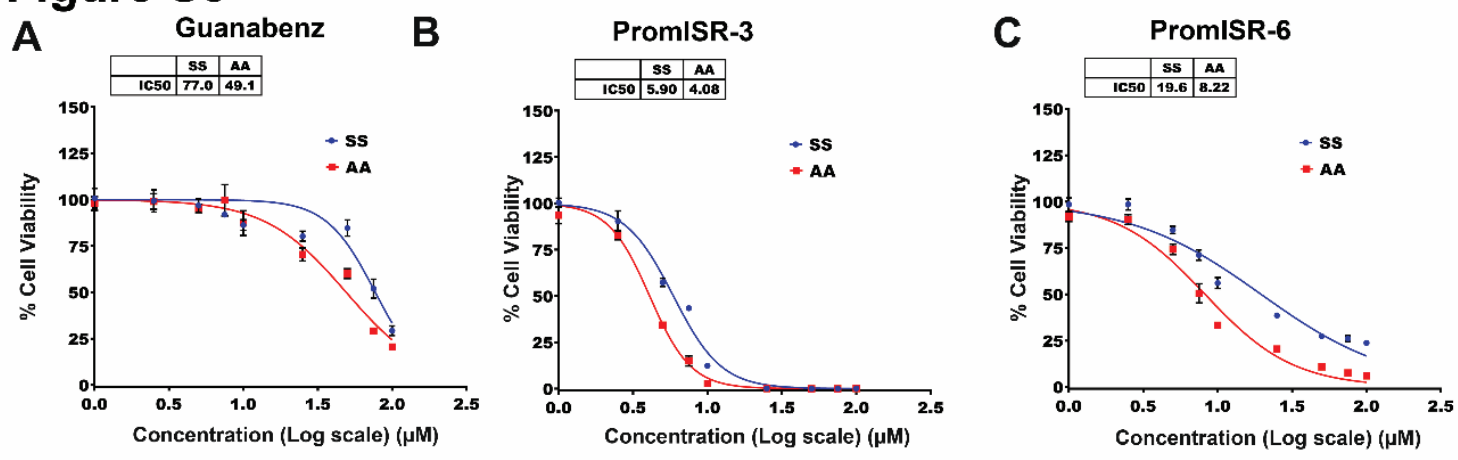

\section{Supplemental Figure S5: Cytotoxicity of SS and AA MEFs with Guanabenz Analogues}

Dose response curves for cytotoxicity of WT or SS and S51A or AA MEFs to Guanabenz (Panel A) PromISR-3 (Panel B) and PromISR-6 (Panel C) (n=3). IC 50 values for each compound with each cell are shown in the boxes. Error bars indicate \pm s.e.m. 


\section{Figure S6}

A
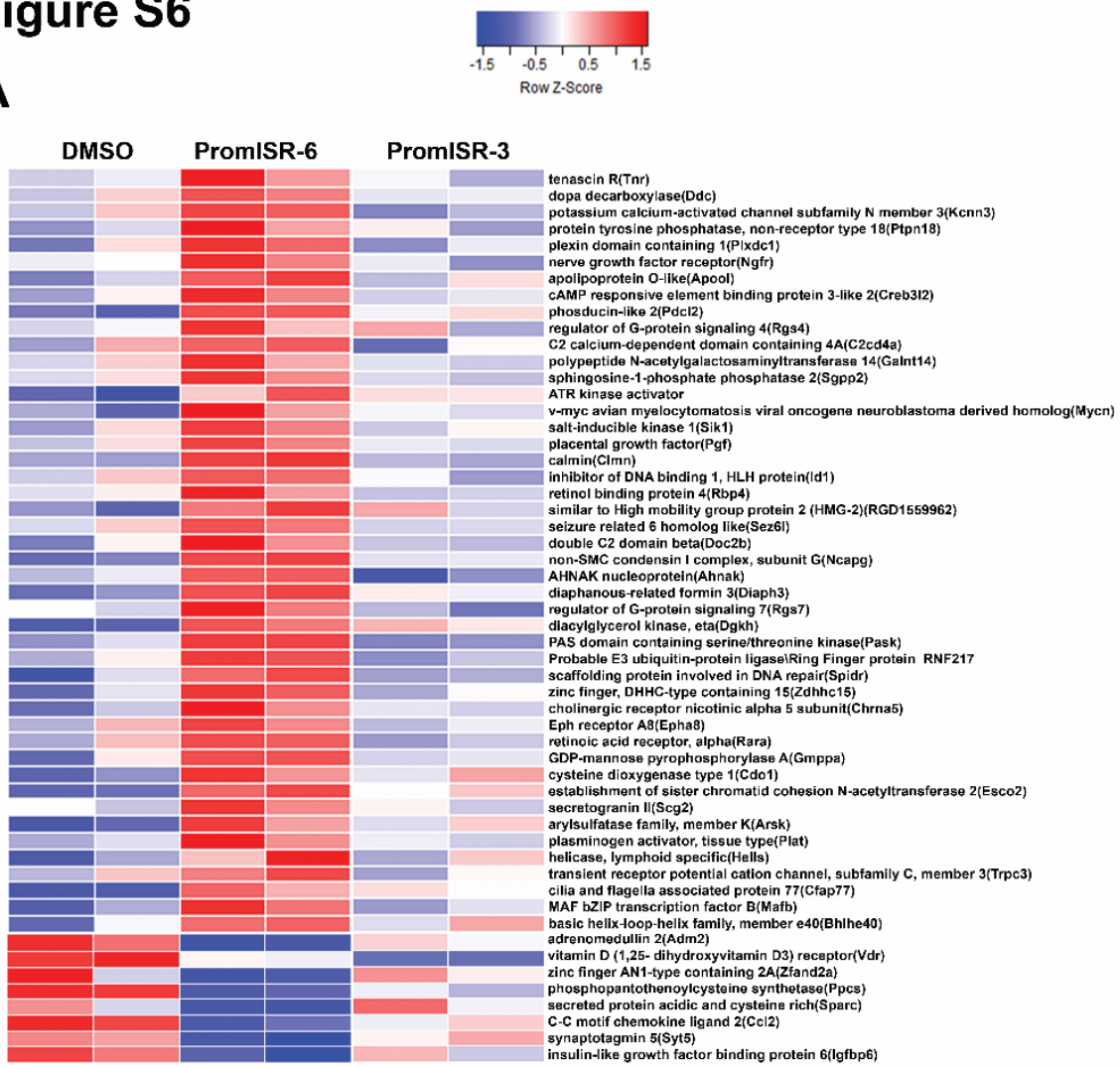

B

Upregulated Pathways

Downregulated Pathways
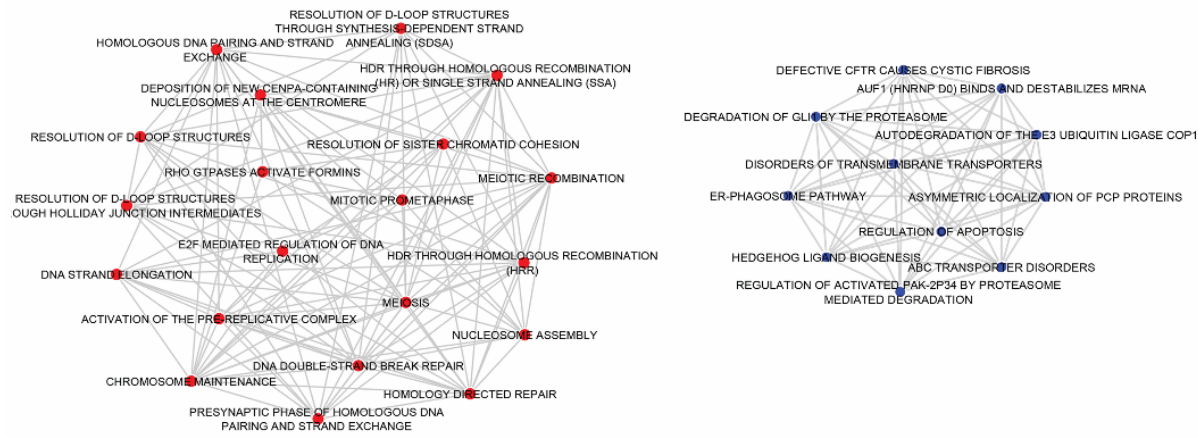

Supplemental Figure S4: Gene Expression Elicited by GBZ analogues in GFP-mHtt-74Qexpressing PC12 Cells

Panel A showing the heatmap of differential gene expression analyzed with Cyber-T using Affymetrix microarray data from GFP-mHtt-74Q-expressing PC12 cells exposed to DMSO, PromISR-6 or PromISR-3 (10 $\mu \mathrm{M})$ for $48 \mathrm{hr}$. Selected genes that were upregulated (red) and downregulated (blue) ( $\geq 1.5$ fold with 1\% FDR) by PromISR-6 and PromISR-3 are shown. Genes, such as tenascin $\mathrm{R}(T N R)$, dopa decarboxylase $(D d c)$, potassium calcium-activated channel subfamily $\mathrm{N}$ member 3 (KCNN3), protein tyrosine phosphatase, non-receptor type 18 (PTPN18), nerve growth factor receptor (NGFR), regulator of G-protein signaling 4 (RGS4), 
seizure related 6 homolog like $(S E Z 6 L)$, diacylglycerol kinase, eta $(D G K H)$, regulator of Gprotein signaling 7 (RGS7) and cholinergic receptor nicotinic alpha 5 subunit (CHRNA5), were previously shown to be downregulated by the expression of mutant Huntingtin ${ }^{4-7}$. The expression of these genes was upregulated or restored by PromISR-6. Panel B shows the networks of upregulated and downregulated biological events, that were deciphered using all genes whose expression was changed by PromISR-6 compared to vehicle and graphically mapped using the gene-set enrichment results from Gene-set-enrichment analysis (GSEA) performed on the Affymetrix microarray data acquired from GFP-mHtt-74Q-expressing PC12 cells. We speculate that the upregulation of DNA repair and replication pathways as well as the cell cycle, which are frequently compromised following UPR activation reflects PromISR6-mediated clearance of mHtt aggregates and cell recovery from the stress associated misfolded mHtt. Consistent with this, pathways that regulated apoptosis, autophagosomes and proteosomal function were significantly downregulated following $48 \mathrm{hr}$ exposure of $\mathrm{mHtt}-$ 74Q-expressing PC12 cells to $10 \mu \mathrm{M}$ PromISR-6.

\section{SUPPLEMENTAL REFERENCES}

1. Irizarry, R. A., Bolstad, B. M., Collin, F., Cope, L. M., Hobbs, B., and Speed, T. P. (2003) Summaries of Affymetrix GeneChip probe level data, Nucleic Acids Res 31, e15.

2. Baldi, P., and Long, A. D. (2001) A Bayesian framework for the analysis of microarray expression data: regularized $t$-test and statistical inferences of gene changes, Bioinformatics 17, 509519.

3. Subramanian, A., Tamayo, P., Mootha, V. K., Mukherjee, S., Ebert, B. L., Gillette, M. A., Paulovich, A., Pomeroy, S. L., Golub, T. R., Lander, E. S., and Mesirov, J. P. (2005) Gene set enrichment analysis: a knowledge-based approach for interpreting genome-wide expression profiles, Proc Natl Acad Sci U S A 102, 15545-15550.

4. Kuhn, A., Goldstein, D. R., Hodges, A., Strand, A. D., Sengstag, T., Kooperberg, C., Becanovic, K., Pouladi, M. A., Sathasivam, K., Cha, J. H., Hannan, A. J., Hayden, M. R., Leavitt, B. R., Dunnett, S. B., Ferrante, R. J., Albin, R., Shelbourne, P., Delorenzi, M., Augood, S. J., Faull, R. L., Olson, J. M., Bates, G. P., Jones, L., and Luthi-Carter, R. (2007) Mutant huntingtin's effects on striatal gene expression in mice recapitulate changes observed in human Huntington's disease brain and do not differ with mutant huntingtin length or wild-type huntingtin dosage, Hum Mol Genet 16, 1845-1861.

5. van Roon-Mom, W. M., Pepers, B. A., t Hoen, P. A., Verwijmeren, C. A., den Dunnen, J. T., Dorsman, J. C., and van Ommen, G. B. (2008) Mutant huntingtin activates Nrf2-responsive genes and impairs dopamine synthesis in a PC12 model of Huntington's disease, $B M C \mathrm{Mol}$ Biol 9, 84.

6. Kusakabe, M., Mangiarini, L., Laywell, E. D., Bates, G. P., Yoshiki, A., Hiraiwa, N., Inoue, J., and Steindler, D. A. (2001) Loss of cortical and thalamic neuronal tenascin-C expression in a transgenic mouse expressing exon 1 of the human Huntington disease gene, $J$ Comp Neurol 430, 485-500. 
7. Hodges, A., Strand, A. D., Aragaki, A. K., Kuhn, A., Sengstag, T., Hughes, G., Elliston, L. A., Hartog, C., Goldstein, D. R., Thu, D., Hollingsworth, Z. R., Collin, F., Synek, B., Holmans, P. A., Young, A. B., Wexler, N. S., Delorenzi, M., Kooperberg, C., Augood, S. J., Faull, R. L., Olson, J. M., Jones, L., and Luthi-Carter, R. (2006) Regional and cellular gene expression changes in human Huntington's disease brain, Hum Mol Genet 15, 965-977. 